\title{
Medicinal flora of the Maranhão, Brazil: plants species and their therapeutic indications in popular use
}

The Brazil is considered a megadiverse country, with a diversified flora, present in their different biomes, with characteristics especially typical, with plants specie with different popular use, major of medicinal utilization. Known the plants species used for the people is important, for very species same not were investigated of scientific form, verifying their potentialities medicinal and therapeutically. The research presented 70 species, distributed in 58 genera and 28 botanical families. The work present for all species with scientific name, family, Common name, part used, use form and Therapeutic indication. Plates with imagens of any plants species medicinal are presents.

\section{Flora medicinal do Maranhão, Brasil: espécies vegetais e suas indicações terapêuticas no uso popular}

O Brasil é considerado um país megadiverso, com uma flora diversificada, presente nos seus diferentes biomas, com características especialmente típicas, com espécies vegetais com diferentes usos populares, principalmente de utilização medicinal. Conhecer as espécies vegetais medicinais usadas pela população e importante, pois muitas espécies ainda não foram investigadas cientificamente, verificando suas potencialidades medicinais e terapêuticas. A pesquisa apresentou 70 espécies, distribuídas em 58 gêneros e 28 famílias botânicas. 0 trabalho apresenta para todas as espécies com nome científico, família, nome popular, parte usada, forma de uso e indicações terapêuticas. Pranchas com imagens de algumas espécies vegetais medicinais são apresentadas.

Palavras-chave: Biodiversidade brasileira; Etnobotânica; Plantas que curam; Vegetação urbana.

Janilde de Melo Nascimento

Universidade Estadual do Maranhão, Brasil http://lattes.cnpq.br/3326215826530556 http://orcid.org/0000-0002-6623-7348 jad-nasci@hotmail.com

Jadson Vinícius Nascimento Oliveira Instituto Federal do Maranhão, Brasil http://lattes.cnpq.br/8937181733563923 http://orcid.org/0000-0001-5658-5608 jadsonvinicruzeiro@gmail.com

Joene Soares da Silva (it)

Instituto Federal do Maranhão, Brasil http://lattes.cnpq.br/7786580983040276 http://orcid.org/0000-0002-2944-2211 joenesoares@live.com

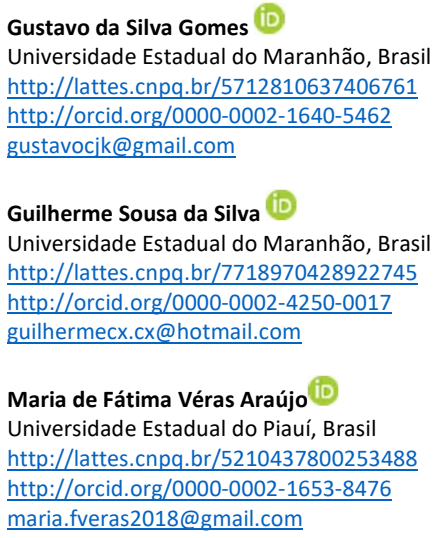

maria.fveras2018@gmail.com

Gonçalo Mendes da Conceição

Universidade Estadual do Maranhão, Brasil http://lattes.cnpq.br/0605946383963749 http://orcid.org/0000-0001-9056-9070

doutorgoncalo@gmail.com d

DOI: 10.6008/CBPC2318-2881.2020.001.0008

\section{Referencing this:}

NASCIMENTO, J. M.; OLIVEIRA, J. V. N.; SILVA, J. S.; GOMES, G. S SILVA, G. S.; ARAÚJO, M. F. V.; CONCEIÇÃO, G. M.. Medicinal flora of the Maranhão, Brazil: plants species and their therapeutic indications in popular use. Nature and Conservation, v.13, n.1, p.61-76, 2020. DOI: http://doi.org/10.6008/CBPC2318-2881.2020.001.0008 


\section{INTRODUCTION}

Brazil is considered one of the most biodiverse countries on the planet, as it has about $20 \%$ of the total number of species in the world (MACIEL et al., 2002; ALBUQUERQUE et al., 2007a). Currently there are an estimated 383,671 species worldwide, where 124,993 species occur on the American continent and 33,161 species are present in the Brazilian territory (ULLOA et al., 2017; LUGHADHA et al., 2016). However, part of this species diversity has not been investigated in relation to their therapeutic potentials, although this is a process that has been evolving significantly (SIMÕES et al., 2003; OLIVEIRA et al., 2009; CARTAXO et al., 2010).

Several cultural groups resort to plants as therapeutic resources, and in recent years their use has intensified as an alternative or complementary to traditional medicine treatments (DORIGONI et al., 2001). The use of medicinal plants is a common practice among peoples around the world and has even received incentives from WHO itself. According to the World Health Organization (WHO), about $80 \%$ of the world's population has used some herb type, seeking relief from some unpleasant symptoms or illness, where at least $30 \%$ of this was due to medical indication (MACIEL et al., 2002).

Medicinal plants are those with therapeutic potential or that have defined effects on diseases or symptoms and, according to Di Stasi (1996), after careful studies, represent an inexhaustible source of commonly approved drugs used, as well as a rich source of new active ingredients, with potential biological activities.

These active ingredients are chemicals resulting from the secondary metabolism of plants that can act beneficially or act toxic to organisms. Therefore, in order for people to be able to safely and safely use these substances, they must be studied from a chemical, pharmacological and toxicological point of view, avoiding indiscriminate use and believing only in empirical knowledge (RITTER et al., 2002).

Many years ago, the accumulated knowledge about medicinal plants was already quite extensive and deep and the healing properties of the plants, their effect on the organism and their application were already known. But the active principles or the reason why the plant acted this way or that in the organism were not known (NARDELLI, 2005).

In this context, further studies with medicinal plants are needed to know and rescue the medicinal potential of vegetables, as well as to provide subsidies for future research and discoveries of new active ingredients. Thus, the research aimed to know the flora of a Cerrado fragment from the state of Maranhão/Brazil, pointing out the medicinal potential of the species found, through bibliographic references, about the used part, form of use and Therapeutic indication of the inventoried species.

\section{MATERIALS AND METHODS}

\section{Study area}

The plant species were collected in the Cerrado fragment existing in the Centro de Estudos Superiores de Caxias/CESC, of the State University of Maranhão/UEMA, which is located in Morro do Alecrim (Figure 
1.A-B-C-D), urban area of the municipality of Caxias/Maranhão/Brazil, among the geographic coordinates, latitude $04^{\circ} 51^{\prime} 93^{\prime \prime}$ South and longitude $43^{\circ} 21^{\prime} 28^{\prime \prime}$ to the west, with an altitude of $275 \mathrm{~m}$, which is the highest point in the city. Its slopes display a beautiful vegetation cover, with typical Cerrado area, with soil, climate and characteristic vegetation.

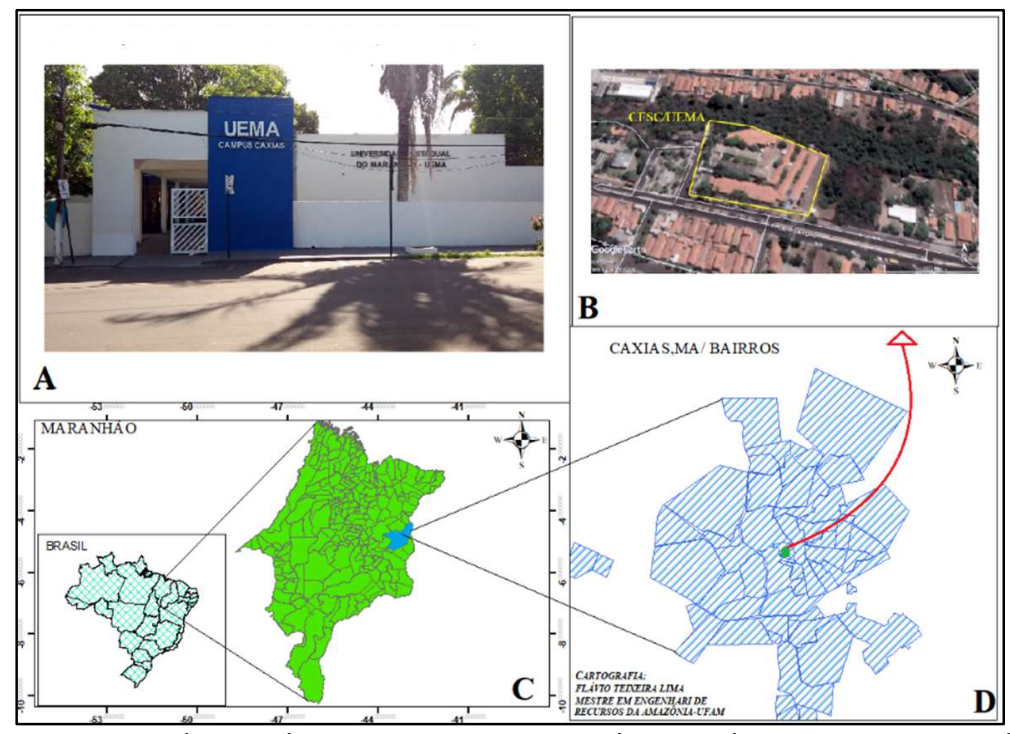

Figure 1: Location map of the CESC/UEMA/ MORRO DO ALECRIM /CAXIAS/MA. A and B. CESC/UEMA Campus. C. Map of Maranhão. D. Map of Caxias with location of Morro do Alecrim/Campus CESC/UEMA. Source: Figure A Authors of the chapter. Figure B Google maps. Figure C and D Flávio Teixeira Lima.

\section{Collect, herborization and identification of the plant species}

The collection of medicinal plants found in the Cerrado fragment, CESC/UEMA, occurred between August 2018 and July 2019 following usual methodology of Fidalgo; Bononi (1989). During the field collections, data on the date, place, habit and other characteristics of the plants were recorded. The identification of the species occurred with the help of specialized literature, identification key and expert botanists. The classification system adopted was APG IV (2016) for Phanerogams and PPG I (2016) for Cryptogams. Subsequently, the material properly herborized and labeled was incorporated into the Herbarium Profo Aluízio Bittencourt/HABIT, Centro de Estudos Superiores de Caxias in State University of Maranhão. Ethnobotanical and medicinal data of the species were informed for each species, following the sequence: scientific name, Common name, part used, forms of use, therapeutic indications and bibliographic sources.

\section{RESULTS AND DISCUSSION}

The research presented 70 species, distributed in 58 genera and 28 botanical families. Among the related groups, only one species of cryptogam was recorded (Lygodium venustum Sw.- Lygodiaceae), while the other species are represented by phanerogamic plant families. The families of phanerogams were distributed according to the number of species: Fabaceae (16 species), Convolvulaceae and Malvaceae (seven species), Euphorbiaceae (six species), Asteraceae and Rubiaceae (three species), while Amaranthaceae, Apocynaceae, Cucurbitaceae., Passifloraceae, Solanaceae and Urticaceae were represented by two species 
each. The families Acanthaceae, Boraginaceae, Commelinaceae, Connaraceae, Lamiaceae, Linderneaceae, Loganiaceae, Nyctaginaceae, Plantaginaceae, Phyllanthaceae, Smilacaceae, Talinaceae, Verbenaceae and Vitaceae each had one species. The most representative genera were: Euphorbia L. (Euphorbiaceae), Ipomoea L. (Convolvulaceae), Mimosa L. (Fabaceae), Sida L. (Malvaceae), with three species each; Altenanthera Forssk. (Amaranthaceae), Croton L. (Euphorbiaceae), Centrosema (DC.) Benth. and Senna Mill. (Fabaceae), with two species each.

\section{Plants species and their Therapeutic indication}

Elytraria imbricata (Vahl) Pers.: Family: Acanthaceae; Common name: Folha da Gentileza; Part used: Root, stem, leaf; Form of use: Decoction; Therapeutic indication: Diarrhea, contra-venom (scorpion bite), kidney pain (PÉREZ et al., 2015).

Alternanthera brasiliana (L.) Kuntze.: Family: Amaranthaceae; Common name: Anador, melhoral, caaponga, cabeça-branca, carrapichinho, quebra-panela, sempre-viva, doril, perpétua-do-brasil, terramicina, sempre-viva; Part used: Whole plant, leaf, flower; Form of use: Infusion, Decoction and Maceration; Therapeutic indication: Bladder, liver, hemorrhoid, pain, antidiarrheal, constipation, diuretics, depurative influenza, cold, sore throat; (SILVA et al., 2011; PLANTAMED, 2018).

Alternanthera tenella Colla.: Family: Amaranthaceae; Common name: Apaga fogo, periquito, corrente, perpétua do campo; Part used: Whole plant; Form of use: Decoction; Therapeutic indication: Headaches and fevers (VENDRUSCOLO et al., 2005; NASCIMENTO, 2014).

Schubertia grandiflora Mart.: Family: Apocynaceae; Common name: Maria-da-costa; Part used: Stem (Tuber); Form of use: Not found; Therapeutic indication: Abortive (SANTOS et al., 2013).

Prestonia bahiensis Müll. Arg.: Family: Apocynaceae; Common name: Cipó cabeludo, gitirana, pau darco; Part used: Flower; Form of use: Not found; Therapeutic indication: Treat kidney problems, back pain, tranquilizer (MORALES, 2017).

Centratherum punctatum Cass.: Family: Asteraceae; Common name: Perpétua-roxa-do-mato, vassourinha-roxa, perpétua-brava-do-mato, perpétua-do-mato, melosa, aletria e suspiro-de-cachorro, contra-veneno, placari; Part used: Leaf; Form of use: Sumo (internal and external); Therapeutic indication: Snake bite (VÁSQUEZ et al., 2014; CARVALHO, 2012).

Cyanthillium cinereum (L.) H. Rob.: Family: Asteraceae; Common name : Erva de ferro, vassourão, trombe; Form of use: Decoction, infusion; Therapeutic indication: Asthma, cancer, cholera, colic, cough, diarrhea, dysentery, impotence, night blindness; anthelmintics, treatment of leprosy, chronic skin diseases, analgesic, antipyretic, scorpion bite antidote and snake, malaria, fever, worms, pain, infections, diuresis, cancer, abortion, gastrointestinal disorder (GUHA et al., 2011; KUMAR et al., 2009).

Tridax procumbens L.: Family: Asteraceae; Common name: Erva-de-touro; Part used: Leaf; Form of use: Decoction, infusion; Therapeutic indication: Anticoagulant, antifungal, insect repellent, phlegm, diarrhea, dysentery, wound healing, promotes hair growth, liver disorders, leishmaniasis (BHAGWAT et al., 2008; CERQUEIRA et al., 2002). 
Heliotropium indicum L.: Family: Boraginaceae; Common name: Crista-de-Galo, cravo-de-urubu, fedegoso, erva-de-são-fiacre; Part used: Root, leaf, flower; Form of use: Decoction; Therapeutic indication: Thrush, stomatitis, throat and pharyngeal ulcerations (LORENZI et al., 2008).

Commelina benghalensis L.: Family: Commelinaceae; Common name: marianinha; Part used: Leaf; Form of use: Decoction; Therapeutic indication: Anticephalic, diuretic (MESSIAS, 2015).

Rourea doniana Baker.: Family: Connaraceae; Common name : Not Found; Part used: Stem, leaf; Form of use: Extract; Therapeutic indication: Antioxidant, promising activity of hexane stem extract against Aedes aegypti larvae, being a potential agent against the dengue-transmitting mosquito, a disease that affects several regions of Brazil (OLIVEIRA et al., 2010; OLIVEIRA, 2011).

Distimake aegyptius (L.) A.R. Simões \& Staples.: Family: Convolvulaceae; Common name: Corda-deviola, batatão roxo, jetirana-de-batata, jetirana-cabeluda, jetirana; Part used: Stem, leaf, seed; Form of use: Extract; Therapeutic indication: Antimicrobial against Escherichia coli, Pseudomonas putida, Bacillus thuringiensis, B. subtilis, Candida albicans and Aspergillus niger, diabetes, wounds, infections and tumors (JOSHI et al., 2015; OMOTAYO et al., 2012).

Evolvulus nummularius (L.) L.: Family: Convolvulaceae; Common name : Aakhukarni, Muusaakarni and Chhinipatra; Part used: Whole plant; Form of use: metabolic extracts; Therapeutic indication: Remedy for hysteria, to cure burns, cuts, wounds and scorpion bites, anti-helminthine, wound healing (PAVITHRA et al., 2009).

Ipomoea asarifolia (Desr.) Roem. \& Schult.: Family: Convolvulaceae; Common name: Salsa, batata de boi; Part used: Stem, leaf, flower; Form of use: Decoction; Therapeutic indication: Leprosy, itch, wound, toothache (Anti-inflammatory) (NETO et al., 2014; FREITAS et al., 2015).

Ipomoea bahiensis Willd. ex Roem. \& Schult.: Family: Convolvulaceae; Common name: Jetirana; Part used: Leaf; Form of use: Extract; Therapeutic indication: Growth inhibition of various microorganisms such as Bacillus subtilis, Staphylococcus aureus, Neurospora crassa, Streptococcus faecalis, anticancer (BIEBER et al., 1986; MEIRA et al., 2012; MAIA-SILVA et al., 2012).

Ipomoea squamosa Choisy.: Family: Convolvulaceae; Common name: Not Known; Part used: Leaf; Form of use: Extract; Therapeutic indication: Has human ovarian cytotoxic activity / tested on ovarian cancer cells / in vitro (CAO et al., 2007).

Jacquemontia tamnifolia (L.) Griseb.: Family: Convolvulaceae; Common name: Corda-de-viola, jetirana; Part used: Root, leaf; Form of use: Crushed foil (poultice), foil powder; leaf juice; Therapeutic indication: Headache, neuralgia, conjunctivitis, snake bite (MOREIRA, 2014).

Operculina hamiltonii (G. Don) D.F. Austin \& Staples.: Family: Convolvulaceae; Common name: Jalapa-brasileira, jalapa, raiz-do-jeticucu, mecoacã, batata-de-purga; Part used: Root, seed; Form of use: Infusion; Therapeutic indication: Digestive tract disorder, amoeba, difficult teething, childhood diarrhea, dysentery, gastroenteritis, haemorrhage, nosebleed, skin disorder, prevent childhood meningitis (PLANTAMED, 2019; BRAGA, 1976).

Cayaponia tayuya (Vell.) Cogn.: Family: Cucurbitaceae; Common name: Taiuiá, tajujá, abobrinha-do- 
mato, cabeça-de-negro; Part used: Root; Form of use: Decoction; Therapeutic indication: Pain in general, blood purifier, purgative, analgesic, erysipelas, rheumatism, ulcers, herpes, boils, analgesics, antiinflammatory (LORENZI et al., 2008).

Momordica charantia L.: Family: Cucurbitaceae; Common name: Melão-de-São-Caetano; Part used: Leaf; Form of use: Leaf extract; Therapeutic indication: Leukorrhea, antidiabetic, healing, antirheumatic, antimicrobial, diabetes, skin lesions (RODRIGUES et al., 2010; DENISE et al., 2009).

Croton hirtus L'Hér.: Family: Euphorbiaceae; Common name: Malva de pelos, croto; Part used: Whole plant; Form (s) of use: Extract; Therapeutic indication: Antibacterial activities (SUBIN et al., 2012).

Croton heliotropiifolius Kunth.: Family: Euphorbiaceae; Common name: Velame or velame-dacaatinga; Part used: Leaf; Form of use: Infusion; Therapeutic indication: Stomachache, stomach upset, vomiting, diarrhea (RANDAU et al., 2004; MARANGONI, 2015).

Euphorbia heterophylla L.: Family: Euphorbiaceae; Common name: Leiteira; Part used: Root, leaf; Form of use: Infusion; Therapeutic indication: Headache, laxatives, anti-diarrhea, migraine, warts, antitumor/anti-cancer and anti-HIV (HILLOCKS, 1998; RODRIGUEZ et al., 1976; FALODUN et al., 2003).

Euphorbia hirta L.: Family: Euphorbiaceae; Common name : Erva-de-Santa-Luzia; Part used: stem (latex), leaf; Form of use: Extract, maceration; Therapeutic indication: Analgesic, anti-inflammatory, antidepressant, antihypertensive, respiratory, gastrointestinal treatment, cough, runny nose, allergic rhinitis, asthma, bronchial infections, worm infestations, kidney stones (TONA et al., 1999, ANJARIA et al., 1997; ELUMALAl, 2010).

Euphorbia prostrata Aiton.: Family: Euphorbiaceae; Common name: Quebra-pedra-rasteira; Part used: whole plant, leaf; Form of use: Decoction; Therapeutic indication: Kidney stones, diarrhea, wound cleaning, urinary tract infection (PLANTAMED, 2018; PILLA et al., 2006; PÉREZ et al., 2015).

Ricinus communis L.: Family: Euphorbiaceae; Common name: Mamona, Carrapateiro, Carrapateiro, Castor, Palma-de-cristo, Rícino, Mamoneira, Tortago Part used: Leaf, seed; Form of use: Seed oil extract (decoction), leaf (poultice); Therapeutic indication: Treatment of rheumatic leg and foot pain, deworming, boils, ear inflammation, laxative, hair growth (LORENZI et al., 2008).

Centrosema brasilianum (L.) Benth.: Family: Fabaceae; Common name: Roxinha, cunhã, Feijãobravo; Part used: Leaf; Form of use: Not found; Therapeutic indication: Astringent, antiseptic (BRANDÃO, 1991; VIEIRA et al., 2000; OLIVEIRA et al., 2015).

Centrosema pubescens Benth.: Family: Fabaceae; Common name: Ervilha borboleta; Part used: Stem, leaf, seed; Form of use: Extract; Therapeutic indication: Wound healing; (NATURE.SCIENCE LIFE, 2019; EKPO et al., 2011).

Chamaecrista desvauxii (Collad.) Killip.: Family: Fabaceae; Common name: Sene do campo; Part used: Leaf, fruit (pod); Form of use: Decoction (tea); Therapeutic indication: Abortion and postpartum lavage (BERG et al., 1988).

Crotalaria retusa L.: Family: Fabaceae; Common name: Amendoim bravo, chique-chique, guiso de cascável; Part used: Seed; Form of use: Flour from seed; Therapeutic indication (s): Anti-inflammatory and 
antinociceptive (reduction in the ability to perceive pain) (ARAGÃO, 2016).

Desmodium triflorum (L) DC.: Family: Fabaceae; Common name: Trevo de carrapato de três-flor, mendigo de três flores; Part used: Whole plant, root, leaf; Form of use: Decoction, poultice; Therapeutic indication: Heat stroke, diarrhea, vomiting, fever, injuries, skin problems, diuretic, mouthwash (FERN et al., 2014; MILKYLANE, 2008; WANG et al., 1994; GAN , 1967; ILANDARA et al., 2015).

Dioclea bicolor Benth.: Family: Fabaceae; Common name: Pucumã; Part used: Seed; Form of use: Sympathies; Therapeutic indication: The use in the pocket to cure cramps (COSTA et al., 2006).

Indigofera suffruticosa Mill.: Family: Fabaceae; Common name: Índigo, anileira; Part used: Root, stem, leaf; Form of use: Infusion, Decoction; Therapeutic indication: Diuretic, abortive, analgesic, against urinary and stomach problems, ulcers, purgative, sedative (PESAVENTO, 2005; MATOS, 1999; HASTINGS, 1990; VIEIRA, 1992; MARTINS, 2000).

Leucaena leucocephala (Lam.) From Wit.: Family: Fabaceae; Common name : Leucaena; Part (s) used: Leaf, seed; Form (s) of use: Extract; Therapeutic indications: Antidiabetic, antibacterial, antihemothetic, cancer preventive, diuretic, nematicide, stomach ache, abortion (ZAYED et al., 2018; DEVI et al., 2013; BREWBAKER et al., 1990; ZAYED et al., 2016; NAS, 1984).

Mimosa arenosa (Willd.) Poir.: Family: Fabaceae; Common name: Unha-de-gato; Part used: Root; Form of use: Lambedor; Therapeutic indication: Influenza, cough (against cough) (SILVA et al., 2015).

Mimosa pudica L.: Family: Fabaceae; Common name: Dormideira, sensitiva, dorme-dorme, não-metoque, malícia, Maria-fecha-a-porta, malícia-roxa; Part used: Whole plant; Form of use: Decoction; Therapeutic indication: Treatment of diabetes, antitoxin, anti-hepatotoxin, antioxidant, wound healing, leprosy, dysentery, vaginal and uterine complaints, inflammation, burning sensation, fatigue, asthma (JOSEPH et al., 2013).

Mimosa sensitiva L.: Family: Fabaceae; Common name: Malícia; Part used: Root; Form of use: Syrup, licking; Therapeutic indication: influenza, cough (against cough) (SILVA et al., 2015).

Desmodium incanum (Sw.) DC.: Family: Fabaceae; Common name: Carrapicho-beiço-de-boi; Part used: Root, leaf; Form of use: Infusion; Therapeutic indication: Antigonorrhea, ovarian problems, asthma, bronchitis, kidney disorders, yellowing, diuretics, kidney problems, blood problems, kidney, bladder, prostate (FLORASBS, 2019).

Senna cana (Nees \& Mart.) H.S. Irwin \& Barneby.: Family: Fabaceae; Common name: São João; Part used: Leaves; Form of use: Extract; Therapeutic indication: In vitro test showed leishmanicidal activities (SARAIVA et al., 2016).

Senna obtusifolia (L.) H.S. Irwin \& Barneby.: Family: Fabaceae; Common name : Mata-pasto; Part used: Root, Leaf, Seed; Form of use: Decoction; Therapeutic indication: Laxative, eye inflammation, dizziness, diarrhea, urinary tract, dysentery, stomach ache, headache (DIRAR, 1984; GUO et al., 1998; ZAFAR, 1994; DOUGHARI et al., 2008 ; ANISUZZAMAN et al., 2014).

Senegalia polyphylla (DC.) Britton \& Rose.: Family: Fabaceae; Common name: Arranha-gato, unha de gato; Part used: Leaf; Form of use: Not found; Therapeutic indication: For fever (DAVID et al., 2015; 
MARREIROS et al., 2015).

Zornia latifolia Sm.: Family: Fabaceae; Common name: Arrozinho, zornia, tencilla; Part used: Leaf, flower, seed (pea); Form of use: Decoction; Therapeutic indication: Inflammation, Relaxation, Sedative, Anxiety (DIXITA et al., 2015).

Marsypianthes chamaedrys (Vahl) Kuntze.: Family: Lamiaceae; Common name : Hortelã-do-campo, erva-do-pacari, hortelã-do-brasil, betônia-brava; Part used: Whole plant, root; Form of use: Decoction; Therapeutic indication: Diarrhea, snake bite, headache, anti-inflammatory, analgesic, carminative, joint rheumatism, anemia (LORENZI et al., 2008).

Linderna crustacea (L.) F. Muell.: Family: Linderneaceae; Common name: Douradinha-do-campo; douradinha-do-Pará, matacana, matucana; orelha-de-rato; Part used: Whole plant; Form of use: Infusion, decoction; Therapeutic indication: Dysentery, diarrhea, vomiting, amenorrhoea, boil, itching, herpes, sores, worms (FERN et al., 2014; PANDA et al., 2011; SOUZA et al., 2009).

Spigelia anthelmia L.: Family: Loganiaceae; Common name: Erva-lombrigueira, lombrigueira ou arapabaca; Part used: Whole plant; Form of use: Infusion, decoction; Therapeutic indication: Prevention and treatment of worm problems (BASÍLIO et al., 2003; GIRÃO et al., 2004; LORENZI et al., 2008).

Lygodium venustum Sw.: Family: Lygodiaceae; Common name: Culebrina, grama de víbora, videira chinesa, crispillo, grama de conversação, ninho de papa, pesma; Part used: Leaf; Form of use: Extract (ethanolic); Therapeutic indication: Antioxidant, antibacterial, antifungal bioactivities, gastrointestinal disorders, infections, dermatoses and parasitic diseases such as trichomoniasis (BRAGA, 2012; TEIXEIRA et al., 2015).

Corchorus aestuans L.: Family: Malvaceae; Common name: Juta; Part used: Root, Leaf, Seed; Form of use: Extract; decoction, powder; Therapeutic indication: Gonorrhea, urethra, headache, fever, stomach problems, anti-inflammatory, pneumonia (N'DANIKOU et al., 2011; KHARE, 2007; AL-SNAFI, 2016).

Melochia pyramidata L.: Family: Malvaceae; Common name: Relógio rosa; Part used: Leaf; Form of use: Decoction; Therapeutic indication: Against cough, bronchitis, urinary tract infection (RAMOS et al., 2018; AGRA et al., 2007; SAMOISY et al., 2016).

Pavonia cancellata (L.) Cav.: Family: Malvaceae; Common name: Malva-rasteira, corda-de-viola; Part used: Leaf; Form of use: Poultice; Therapeutic indication: Boil (AGRA et al., 2007).

Sida acuta Burm.f.: Family: Malvaceae; Common name: Malva-baixa, guaxuma, relógio, tupiticha, vassoura, vassourinha; Part used: Whole plant; Form of use: Infusion, extraction; Therapeutic indication: Fever, headache, skin disease, diarrhea, dysentery (FLORASBS, 2019; KAROU et al., 2007; KUMAR et al., 2013).

Sida ciliaris L.: Family: Malvaceae; Common name: Vassoura; Part used: Whole plant; Form of use: Decoction, infusion; Therapeutic indication: Expectorant and anti-inflammatory (CARMONA et al., 2008).

Sida spinosa L.: Family: Malvaceae; Common name: Guanxuma-de-espinho, guanxuma, malvinha, malva-lanceta, vassourinha-de-relógio; Part used: Whole plant, root, leaf, fruit; Form of use: Decoction and infusion; Therapeutic indication: Ulcers, pain, asthma, burning sensation, skin disease, snake bite, gonorrhea, 
diarrhea, dysentery (FLORASBS, 2019; SHARMA, 2018).

Waltheria indica L.: Family: Malvaceae; Common name: Malva-branca, malva-veludo; Part used: Whole plant; Form of use: Decoction or infusion; Therapeutic indication: Dysentery, catarrh-bronchial, lung disorders, bladder inflammation, cough, bronchitis, syphilis, wound care (LORENZI et al., 2008).

Boerhavia diffusa L.: Family: Nyctaginaceae; Common name: Pega-pinto, agarra-pinto, bredo-deporco, erva-de-porco, batata-de-porco, tangaracá, erva-tostão; Part used: Whole plant, root; Form of use: Decoction, poultice; Therapeutic indication: Hepatitis, jaundice, gallstones, kidneys, diuretics, snake bite, liver (LORENZI and MATOS, 2008).

Passiflora glandulosa Cav.: Family: Passifloraceae; Common name: Maracujá-do-mato; Part used: Leaf, fruit, seed; Form of use: Consumption in natura (pulp extract); Therapeutic indication: Sources of antioxidants may have hypoglycemic effect (LIMA-NETO et al., 2017).

Turnera subulata Sm.: Family: Passifloraceae; Common name: Chanana; Part used: Root, leaf, flower; Form of use: Infusion, edible plant; Therapeutic indication: Liver, anti-inflammatory, against Acquired Immunodeficiency Syndrome (AIDS) (MADALENO, 2011; KINUPP et al., 2014).

Scoparia dulcis L.: Family: Plantaginaceae; Common name: Vassourinha-de-botão, vassourinha-doce, vassourinha-mofina, vassourinha; Part used: Whole plant; Form of use: Decoction, infusion, juice; Therapeutic indication: Fever, cough, bronchitis, diarrhea, inflammation, toothache, stomach, hemorrhoids, insect bites, antidiabetic (LORENZI et al., 2008).

Phyllanthus niruri L.: Family: Phyllanthaceae; Common name: Quebra-pedra, arrebenta-pedra, ervapombinha Part used: Whole plant; Form of use: Decoction or infusion; Therapeutic indication: Urinary tract (renal calculus), fight against hepatitis B virus, assist in the elimination of uric acid (LORENZI et al., 2008).

Bredemeyera floribunda Willd.: Family: Polygalaceae; Common name: Pau-caixão, pau-gemada, cabão-de-bugre, laça-vaqueiro, marfim-de-rama, pau-rendoso, raiz-de-cobra, raiz-de-são-joão-da-costa;Part used: Root; Form of use: Tincture, decoction, infusion; Therapeutical indication: Anti-allergic, antiinflammatory action against bee stings, spiders, scorpions, irritation by stinging plants, dermatitis, catarrhal bronchitis, boils (MARONI et al., 2006; LORENZI et al., 2008).

Borreria verticillata (L.) G. Mey.: Family: Rubiaceae; Common name: Cordãozinho-de-frade, ervabotão, erva-de-lagarto, falsa-poaia, perpétua-do-mato, poaia, poaia-comprida, poaia-falsa, poaia-preta, poaia-rosário, vassoura-botão, vassourinha Part used: Root, leaf; Form of use: Infusion, decoction, poultice or plaster (for burns); Therapeutic indication: Vomiting, diuretic, childhood diarrhea, hemorrhoids, varicose veins, burns (LORENZI et al., 2008).

Richardia grandiflora (Cham. \& Schltdl.) Steud.: Family: Rubiaceae; Common name: Poaia-rasteira, poaia-da-praia, poaia-rósea, asa-de-pato, ipema-mirim, poaia, ipecacuanha-do-campo, ervanço; Part used: Root; Form of use: Decoction; Therapeutic indication: Bronchitis, phlegm, diarrhea and teething, hemorrhoids vermicide (SOUZA et al., 2013).

Oldenlandia corymbosa L.: Family: Rubiaceae; Common name: Erva- diamante; Part used: Whole plant; Form of use: poultice, decoction and juice; Therapeutic indication: Activate blood circulation, promote 
diuresis, relieve stranguria (urinary obstruction), digestive tract lymphosarcoma tumors, liver and larynx carcinoma. It is also active against appendicitis, hepatitis, pneumonia, cholecysthesis, urinary tract infection, cellulitis and snake bite (PATEL et al., 2014).

Smilax cissoides Mart. Ex Griseb.: Family: Smilacaceae; Common name : Sarsaparilla, japecanga; Part used: Stem, leaf; Form of use: Decoction; Therapeutic indication: Diuretic, diaphoretic, apperetic, eupeptic, emollient, expectorant, antileprous, myotonic, sudorific, treatment of syphilis, against eczema, wart, boils, rheumatism, arthritis, vesicular and renal disorders (MEDEIROS et al., 2007; NARDELLI, 2005).

Physalis angulata L.: Family: Solanaceae; Common name: Camapu, camapum, joá-de-pacote, balão; Part used: Root, Leaf, Fruit; Form of use: Infusion, decoction; Therapeutic indication: Anti-inflammatory, skin disease disinfectant, diabetes, chronic rheumatism, kidney problems, bladder, liver, sedative, antifebrile, anti-inflammatory, cytotoxic action for various types of cancer cells, antiviral activity, including against HIV and HSV-1 causing cold sores (LORENZI et al., 2008).

Solanum Iycocarpum A.St.-Hil.: Family: Solanaceae; Common name: Fruta-de-lobo, berinjela-domato, jurubebão, baba-de-boi, capoeira-branca, lobeira; Part used: Leaf, flower, fruit; Form of use: Decoction, fruit juice; Therapeutic indication: Diuretic, soothing, antiepileptic, urinary tract infection, abdominal and renal colic and hemorrhoids, diabetes, cholesterol lowering, epilepsy, asthma, flu, cold (LORENZI et al., 2008; MARONI et al., 2006).

Talinum fruticosum (L.) Juss.: Family: Talinaceae; Common name: João-gomes, folha d'água; Part used: Aerial part (stem, leaf, flower, fruit, seed); Form of use: Extract; Therapeutic indication: Polyuria, internal heat, measles, anti-inflammatory, gastrointestinal disorders (CHAN et al., 2016).

Cecropia pachystachya Trécul.: Family: Urticaceae; Common name: Embaúba, pau-de-lixa; Part used: Stem marrow, leaf; Form of use: Decoction, Natural sap of the stem; Therapeutic indication: Kidney, anemia, anti-inflammatory, antihypertensive stones (BIRTH et al., 2011; LORENZI et al., 2008).

Laportea aestuans (L.) Chew.: Family: Urticaceae; Common name: Urtiga, urtiga vermelha, cansanção Part used: Leaf; Form of use: Decoction; Therapeutic indication: Diabetes mellitus, reproductive problems such as infertility, menstruation, shortening labor, anemia, fibroids, dermatitis, low calcium (LANS et al., 2006; LANS et al., 2007; JIOFACK et al., 2009).

Lantana camara L.: Family: Verbenaceae; Common name : Camará-de-cheiro e camará de chumbo; Part used: Root, leaf, flower; Form of use: Decoction, infusion; Therapeutic indication: Treatment of itching, stomachache, toothache, rheumatism, cutting, flu, asthma, bronchitis and antiseptic, for bathing, antimalarial, antimicrobial (fungal and bacterial) scabies (BEZERRA et al., 2016; LORENZI et al., 2008; MARONI et al., 2006).

Cissus verticillata (L.) Nicolson \& C.E. Jarvis.: Family: Vitaceae; Common name: Insulina-vegetal; uvinha, uva-brava; Puçá; Part used: Root, stem, leaf; Form of use: Juice, infusion; Therapeutic indication: Heart problems, anemia, rheumatism, anticonvulsant, antidiabetic, intestine (CORRÊA, 1926; ALMEIDA et al., 1994; SILVA et al., 1994; POTT et al., 1994; LORENZI et al., 2008; SOUZA et al., 2009). 


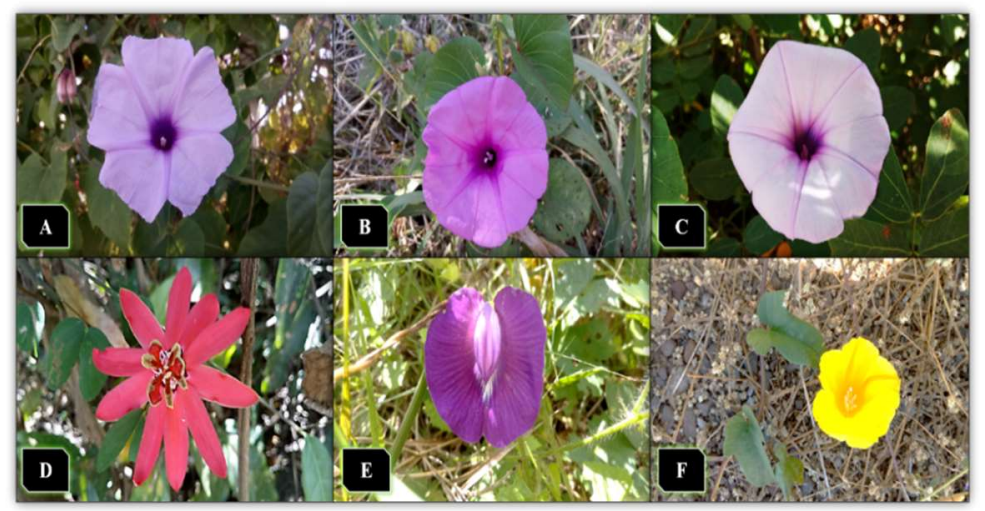

Figure 2: Plant species found in Morro do Alecrim/Caxias/MA A - Ipomoea bahiensis Willd. ex Roem. \& Schult. B Ipomoea asarifolia (Desr.) Roem. \& Schult. C - Ipomoea squamosa Choisy. D - Passiflora glandulosa Cav. E -

Centrosema brasilianum (L.) Benth. F- Operculina hamiltonii (G. Don) D.F. Austin \& Staples.

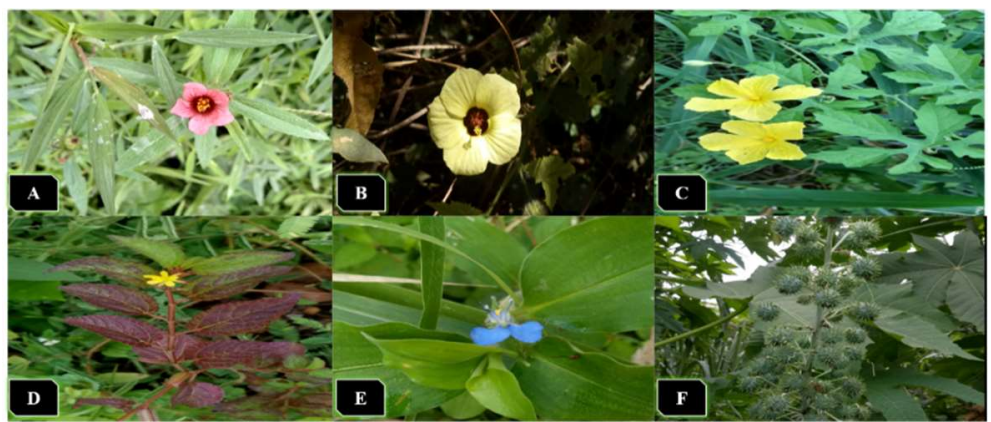

Figure 3: Plant species found in Morro do Alecrim/Caxias/MA. A - Sida ciliares L. (A). B - Pavonia cancellata (L.) Cav. C Momordica charantia L. D - Corchorus aestuans L. E -Commelina benghalensis L. F - Ricinus communis L.

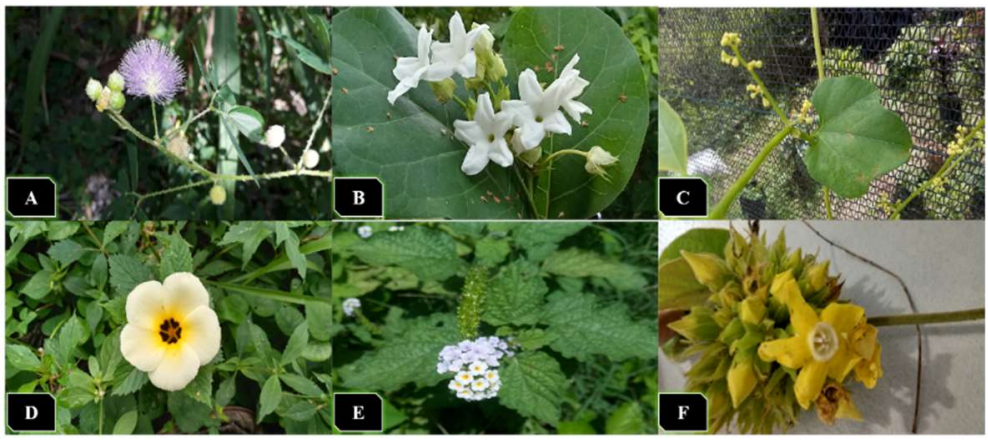

Figure 4: Plant species found in Morro do Alecrim/Caxias/MA. A - Mimosa sensitiva L.; B - Schubertia grandiflora Mart. C - Cayaponia tayuya (Vell.) Cogn. D - Turnera subulata Sm. E-Heliotropium indicum L. F - Prestonia bahiensis Müll.Arg.

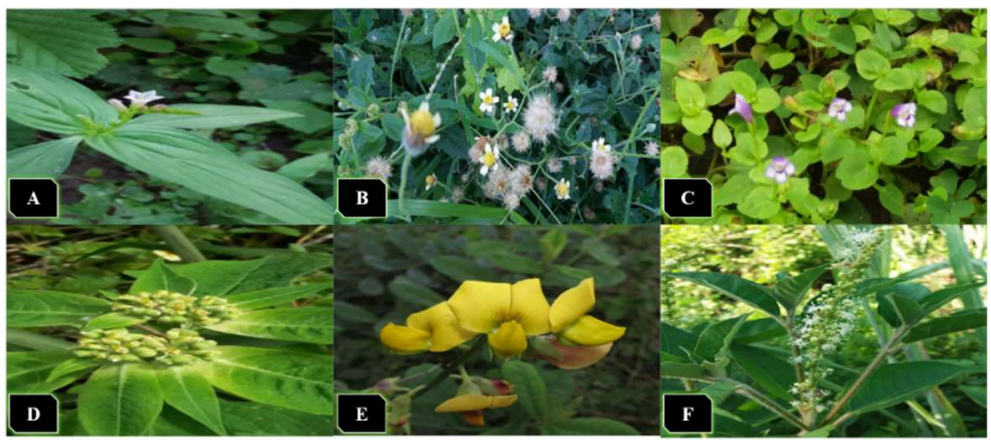

Figure 5: Plant species found in Morro do Alecrim/Caxias/MA. A - Spigelia anthelmia L. B - Tridax procumbens L. C Linderna crustacea. D - Euphorbia heterophylla L. E - Crotalaria retusa L. F - Croton heliotropiifolius Kunth.

\section{CONCLUSIONS}

The research contributed to increase the number of plant species, with medicinal information in Maranhão/Brazil, where many species were indicated as useful for the treatment of common diseases such 
as influenzas, urinary infections, headaches, dysentery, among others. The knowledge and indication of these medicinal plants to cure diseases are important tools in the discovery of new drugs, as well as being the basis for new research.

Despite the various medicinal and ethnobotanical knowledge, there are still many plant species that have no phytochemical studies. Thus, it is important to mention that there is a need for further studies of this nature, for the knowledge of medicinal plants, and thus, to verify their efficiency to cure diseases, since some plants may have secondary metabolites that may be toxic. humans when used in large quantities. Even so, it is worth emphasizing the importance of preserving urban plant fragments and the researched area, to ensure plant diversity, with medicinal purposes, as well as being subsidies for future research on the medicinal flora of Maranhão/Brazil.

\section{REFERENCES}

AGRA, M. F.; BARACHO, G. S.; NURIT, K.; BASÍLIO, I. J. L. D.; COELHO, V. P. M.. Medicinal and poisonous diversity of the flora of "Cariri Paraibano", Brazil. Journal of Ethnopharmacology. v.111, p.383-395, 2007. DOI: https://10.1016/j.jep.2006.12.007

APG IV. An update of the Angiosperm Phylogeny Group classification for the orders and families of flowering plants: APG IV. Botanical Journal of the Linnean Society, v.181, p.120, 2016. DOI: https://doi.org/10.1111/boj.12385

ALBUQUERQUE, U. P.; MEDEIROS, P. M.; ALMEIDA, A. L.; MONTEIRO, J. M.; LINS NETO, E. M. F.; MELO, J. G.; SANTOS, J. P.. Medicinal plants of the caatinga (semi-arid) vegetation of NE Brazil: A quantitative approach. Journal of Ethnopharmacology, v.114, p.325-354, 2007. DOI: https://10.1016/i.jep.2007.08.017

ALMEIDA, M. I. G.; SILVA, G. A.; FERRO, V. O.. Carotenóides em Cissus sicyoides (L.) In: SIMPÓSIO DE PLANTAS MEDICINAIS DO BRASIL, 13. Anais. Fortaleza, 1994.

ALMEIDA, C. F. C. B. R.; ALBUQUERQUE, U. P.. Uso e conservação de plantas e animais medicinais no estado de Pernambuco (Nordeste do Brasil): Um estudo de caso. Interciência, V.27, n.6, p.276-285, 2002.

AL-SNAFI, A. E.. The Constituents and Pharmacology of Corchorus aestuans: A Review. The Pharmaceutical and Chemical Journal. v.3, n.4, p.208-214, 2016.

ANISUZZAMAN, M. D.; AHSAN, Q. M. D.; KUDDUS, R. M. D.; RASHID, M. A.. Pharmacological Activities of Senna obtusifolia Linn.: A Medicinal Plant of Bangladesh. Bangladesh Pharmaceutical Journal, v.17, n.2, p.182-186, 2014. DOI: http://dx.doi.org/10.3329/bpj.v17i2.22338

ANJARIA, J.; PARABIA, M.; BHATT, G.; KHAMAR, R.. Nature heals: a glossary of selected indigenous medicinal plants of India. India7 Sristi Innovations, Ahmedabad, p.20, 1997.

ARAGÃO, D. P.. Atividade anti-inflamatória e antinociceptiva de frações proteicas de sementes de Crotalaria retusa. Dissertação (Mestrado em Ciências Biomédicas) - Universidade Federal do Piauí, Teresina, 2016.

BANI, S.; KAUL, A.; KHAN, B.; GUPTA, V. K.; SATTI, N. K.; SURI, K. A.. Antiarthritic activity of a polymeric fraction from Euphorbia tirucalli. Journal of Ethnopharm, v.37, n.4, p.333336, 2016. DOI: http://10.1016/j.jep.2006.09.021
BASÍLIO, I. J. L. D.; NURIT, K. G. S.; BARACHO, A. M. F.. Caracterização Morfo-Anatômica de Spigelia anthelmia L. (Loganiaceae), espécie da Medicina popular na Paraíba, Brasil. Revista Nordestina de Biologia, v.17, n.1-2, p.11-22, 2003.

BENNETT, B. C.; PRANCE, G. T.. Introduced plants in the indigenous pharmacopoeia of Northern South America. Economic Botany, v.54, n.1, p.90-102, 2000.

BERG, M. E.; VAN DEN SILVA, M. H. L.. Contribuição à flor a medicinal de Mato Grosso do Sul. Supl. Acta Amazônica, v.18, n.1-2, p.9-22, 1998.

BEZERRA, J. W. A.; COSTA, A. R.; RODRIGUES, F. C.; DUARTE, A. E.; ROCHA, M. I.; BARROS, L. M.. Potencial medicinal de Lantana camara L. (VERBENACEAE): Uma revisão. Universidade Regional do Cariri. URCA Caderno de Cultura e Ciência, v.15, n.1, 2016

BHAGWAT, D. A.; KILLEDAR, S. G.; ADNAIK, R. S.. Antidiabetic activity of leaf extract of Tridax procumbens. International Journal of Green Pharmacy, v.2, n.2, p.126128, 2008.

BIEBER, L. W.; SILVA FILHO, A. A.; LIMA, R. M. O. C.; CHIAPPETA, A. A.; NASCIMENTO, S. C.; SOUZA, I. A.; MÉLLO, J. F.; VEITH, H. J.. Anticancer and antimicrobial glycosides from Ipomoea bahiensis. Phytochemistry, v.25, p.10771081, 1986.

BRAGA, M. F. B. M.. Prospecção Química do extrato etanólico das folhas de Lygodium venustum SW (Lygodiaceae) e avaliação das bioatividades antioxidante, antiepimastigota, antipromastigota, citotóxica e antimicrobiana de extrato e frações (in vitro). Dissertação (Mestrado em Bioprospecção Molecular) - Universidade Regional do Cariri, Crato, 2012.

BRAGA, R.. Plantas do Nordeste, especialmente do Ceará. 3 ed. Fortaleza, 1976.

BRANDÃO, M.. Plantas medicamentosas do cerrado mineiro. Informe Agropecuário, v.15, n.168, p.15-20, 1991.

BREWBAKER, J.; SORENSSON, C. T.. New tree crops from interspecific Leucaena hybrids. In: JANICK, J.; SIMON, J. E.. Advances in new crops. Portland: Timber Press, 1990. p.2839.

CAPELLARI JUNIOR, L.; NASCIMENTO, C. R.. Guia de Plantas 
Medicinais, aromáticas e condimentares, 200 espécies. Piracicaba, 2011.

CAO, S.; NORRIS, A.; WISSE, J. H.; MILLER, J. S.; EVANS, R.; KINGSTON, D. G.. Ipomoeassin F, a new cytotoxic macrocyclic glycoresin from the leaves of Ipomoea squamosa from the Suriname rainforest. Nat Prod Res., v.21, p.872-876, 2007. DOI: http://10.1080/14786410600929576

JUAN, C. A.; RICARDO, G. O.; CONCEPCION, M. A. R. Descripción taxonómica, morfológica y etnobotánica de 26 hierbas comunes que crecen en la ciudad de Mérida/Venezuela. Boletín Antropológico, v.26, n.7, p.113129, 2008

CARTAXO, S. L.; SOUZA, M. M. A.; ALBUQUERQUE, U. P.. Medicinal plants with bioprospecting potential used in semiarid northeastern Brazil. Journal of Ethnopharmacology, v.131, p.326-342, 2010.

CARVALHO, D. F.. Estudo do potencial antioxidante in vitro e in vivo do extrato aquoso das folhas de Centratherum punctatum ssp punctatum Cass. (Asteraceae). Dissertação (Mestrado em Ciências Farmacêuticas) - Universidade Federal do Piauí, Teresina, 2011.

CERQUEIRA, E. S.; PINTO, J. E. B. P.; MORAIS, A. R.; CASTRO, N. E. A.; CARDOSO, M. G.; LAMEIRA, O. A.. Indução de calos em erva-de-touro (Tridax procumbens L.) utilizando diferentes reguladores de crescimento e tipos de explantes. Ciênc. agrotec., Lavras, v.26, n.2, p.301-308, 2002.

CHAN, Y. S.; KHOO, K. S.; SIT, N. W.. Investigation of twenty selected medicinal plants from Malaysia for antiChikungunya virus activity. International Microbiology, v.19, n.3, p.175-182, 2016. DOI: http://10.2436/20.1501.01.275

CORRÊA, M. P. I. O.. Dicionário das Plantas Úteis do Brasil e das Exóticas Cultivadas. Rio de Janeiro: Imprensa Nacional, 1926.

COSTA, L. C. B.; ROCHA, E. A.; SILVA, L. A. M.; JARDIM, J. G.; SILVA, D. C.; GAIÃO, L. O.; MOREIRA, R. C. T.. Levantamento Preliminar das Espécies Vegetais com Potencial Econômico no Parque Municipal da Boa Esperança, Ilhéus, Bahia, Brasil. Acta Farm., Bonaerense, v.25, n.2, p.184-91, 2006.

DAVID, M.; GONÇALVES, K. G.; NETO, G. G.. A Subfamília Mimosoideae (Fabaceae) para a flora de Mato Grosso, Brasil. Biodiversidade, v.14, n.3. p.19, 2015.

COUTINHO, D. F.; FLORÊNCIO, J. C.; AGUIAR, L. R.; RODRIGUES, K. A. F.; VILANOVA, C. M.; BORBA, E. R. C. Estudo farmacobotânico das folhas de Momordica charantia L. (Cucurbitaceae). Visão Acadêmica, Curitiba, v.10, n.1, p.711, 2009. DOI: http://dx.doi.org/10.5380/acd.v10i1.21314

DEVI, V. M.; ARIHARAN, V. N.; PRASAD, P. N.. Nutritive value and potential uses of Leucaena Leucocephala as Biofuel-a mini review. Res J Pharm Biol Chem Sci, v.4, p.515-21, 2013.

DI STASI, L. C.. Plantas medicinais: arte e ciência. São Paulo: UNESP, 1996.

DIRAR, H.. Kawal, meat substitute from fermented Cassia obtusifolia leaves. Economy Botany, Springer New York, v.38, p.342-349, 1994.

DIXITA, A. K.; CHAURASIAB, B.. Ethno-medicinal Uses of weeds of Guru Ghasidas. Central University, Bilaspur, (CG) India. The Journal of Ethnobiology and Traditional Medicine, v.125, p.1046-1054, 2015.

DORIGONI, P. A.; GHEDINI, P. C.; FRÓES, L. F.; BAPTISTA, K. C.; ETHUR, A. B. M.; BALDISSEROTTO, B.; BÜRGER, M. E.;
ALMEIDA, C. E.; LOPES, A. M.; ZACHIA, R. A.. Levantamento de dados sobre plantas medicinais de uso popular no município de São João do Polêsine, RS, Brasil. I - Relação entre enfermidades e espécies utilizadas. Revista Brasileira de Plantas Medicinais, v.4, n.1, p.69-79, 2001.

DOUGHARI, J. H.; EL-MAHMOOD, A. M.; TYOYINA, I. Antimicrobial activity of leaf extracts of Senna obtusifolia (L). African J. Pharm.Pharmacol., v.2, p.7-13, 2008.

EKPO, M.; MBAGWU, H.; JACKSON, C.; ENO, M. Antimicrobial and Wound Healing activities of Centrosema Pubescens (Leguminosae). JPCS, v.6, p.1-6, 2011.

ELUMALAI, E. K.; PRASAD, T. N. V. K. V.; HEMACHANDRAN, J.; THERASA, S. V.; THIRUMALAI, T.; DAVID, E.. Extracellular synthesis of silver nanoparticles using leaves of Euphorbia hirta and their antibacterial activities. Journal of Pharmaceutical Sciences and Research, v.2, n.9, p.549-554, 2010.

FALODUN, A.; AGBAKWURU, E. O. P.; UKOH G. C. Antibacterial Activity of Euphorbia heterophylla Linn (Family Euphorbiaceae). Pak. Pakistan journal of scientific and industrial research, v.46, n.6, p.471- 472, 2003.

FERN, K.; FERN, A.. Useful Tropical Plants. 2014.

FIDALGO, O.; BONONI, V. L. R.. Técnicas de coleta preservação e herborização de material botânico. São Paulo: Instituto de Botânica, 1989.

FREITAS, A. V. L.; COELHO, M. F. B.; PEREIRA, Y. B.; FREITAS NETO, E. C.; AZEVEDO, R. A. B.. Diversidade e usos de plantas medicinais nos quintais da comunidade de São João da Várzea em Mossoró, RN. Revista Brasileira de Plantas Medicinais, Campinas, v.17, n.4, p.845-856, 2015.

GAN, W. S.. Tanwang Medicinal Flora. Taibei: National Research Institute of Chinese Medicine, 1967.

GIRÃO, E. S.; CARVALHO, J. H.; LEAL, T. M.; VIEIRA, L. S.; MEDEIROS, L. P.. Plantas medicinais no controle de helmintos em caprinos. Teresina: Embrapa, 2004.

GUHA, G.; RAJKUMAR, V.; KUMAR, R. S.; MATHEW, L. Therapeutic Potential of Polar and Non-Polar Extracts of Cyanthillium cinereum In Vitro. Hindawi Publishing Corporation, v.54, n.10, p.1-10, 2011. DOI: https://10.1093/ecam/nep155

GUO, H.; CHANG, Z.; YANG, R.; GUO, D.; ZHENG, J. Anthraquinones from hairy root cultures of Cassia obtusifolia. Phytochemistry, v.49, p.1623-1625, 1998.

HASTINGS, R. B.. Medicinal legumes of Mexico: Fabaceae, Papilionoideae, part one. Economic Botany, v.44, p.336-348, 1990.

HILLOCKS, R. J.. The potential benefits of weeds with reference to small holder agriculture in Africa. Integrated Pest Management Reviews, v.3, p.155-167, 1998.

ILANDARA, R.; CHANDRAPALA, R.; JAYASURIYA, W. J. A. B. N.; SURESH, T. S.. Phytochemical and ethno-pharmacological properties of Desmodium triflorum: A Review. Pharmaceutical Journal of Sri Lanka, p.34-38, 2015.

JIOFACK, T.; FOKUNANG, C.; GUEDJE, N.; KEMEUZE, V.. Ethnobotanical uses of some plants of two ethnoecological regions of Cameroon. Afr J Pharm Pharacol, v.3, p.664-684, 2009.

JOSEPH, B.; GEORGE, J.; MOHAN, J.. Pharmacology and Traditional Uses of Mimosa pudica. International Journal of 
Pharmaceutical Sciences and Drug Research, v.5, n.2, p.4144, 2013.

JOSHI, R.; AGARWAL, S.; PATNI, V.. Evaluation of AntiMicrobial Activity of in vitro and in Vivo Plant Parts of Merremia Dissecta and Merremia aegyptia. IJPSR, v.6, n.6, p.2477-2483, 2015.

KAROU, S. D.; NADEMBEGA, W. M. C.; DENISE, P.; ILBOUDO, D. P.; DJENEBA OUERMI, D.; GBEASSOR, M.; SOUZA, C.; SIMPORE, J.. Sida acuta Burm. f.: a medicinal plant with numerous potencies. African Journal of Biotechnology, v.6, n.25, p.2953-2959, 2007.

KHARE, C. P.. Indian medicinal plants, an illustrated dictionary. Springer Science and Business Media, 2007.

KINUPP, V. F.; LORENZI, H.. Plantas Alimentícias NãoConvencionais (PANC) no Brasil: guia de identificação, aspectos nutricionais e receitas ilustradas. Nova Odessa: Plantarum, 2014.

KUMAR, P. D.; KUMAR, P. A.; KANTA, B. R.; SHIVESH, J.; RANJAN, M. M.; ASHUTOSH, M.; SANJAY, C.. Ethno medicinal and therapeutic potential of Sida acuta. International Research Journal of Pharmacy, v.4, n.1, p.88-92, 2013.

KUMAR, P. P.; KUTTAN, G.. Vernonia cinerea L. Scavenges free radicals and regulates nitric oxide and proinflammatory cytokines profile in carrageenan induced paw edema model. Immunopharmacology and Immunotoxicology, v.31, n.1, p.94-102, 2009.

LANS, C.. Ethnomedicines used in Trinidad and Tobago for reproductive problems. Journal of Ethnobiology and Ethnomedicine, v.3, n.13, p.1-12, 2007.

LANS, C. A.. Ethnomedicines used in Trinidad and Tobago for urinary problems and diabetes mellitus. Journal of Ethnobiology and Ethnomedicine, v.13, n.2, p.45, 2006.

LIMA-NETO, A. B. M.. Antioxidant activity and physicochemical analysis of passion fruit (Passiflora glandulosa Cav.) pulp native to Cariri region. Acta Scientiarum, Biological Sciences Maringá, v.39, n.4, p.417422, 2017.

LORENZI, H.; MATOS, F. J. A., Plantas medicinais no Brasil: nativas e exóticas. 2 ed. Nova Odessa: Plantarum, 2008.

LUGHADHA, E. N.; GOVAERTS, R.; BELYAEVA, I.; BLACK, N.; LINDON, H.; ALLKIN, R.; MAGILL, R. E.; NICOLSON, N.. Counting counts: revised estimates of numbers of accepted species of flowering plants, seed plants, vascular plants and land plants with a review of other recent estimates. Phytotaxa, v.272, p.82-88, 2016.

MACIEL, M. A. M.; PINTO, A. C.; VEIGA, JR V.F.; GRYNBERG, N. F.; ECHEVARRIA, A.. Plantas Medicinais: A Necessidade de Estudos Multidisciplinares. Química Nova, v.25, n.3, p.429438, 2002.

MADALENO, I. M.. Plantas da medicina popular de São Luís, Brasil. Boletim do Museu Paraense Emílio Goeldi. Cienc. Hum., Belém, v.6, n.2, p.273-286, 2011.

MAIA-SILVA, C.; SILVA, C. I.; HRNCIR, M.; QUEIROZ, R. T.; IMPERATRIZ-FONSECA, V. L.. Guia de plantas visitadas por abelhas na Caatinga. Fortaleza: Fundação Brasil Cidadão, 2012.

MARANGONI, C.. Plantas Medicinais Tradicionalmente Utilizadas no Nordeste do Brasil: Potencial Antimicrobiano para Tratar Distúrbios das vias Gênito-Urinárias. Dissertação (Mestrado Ciências Biológicas) - Universidade Federal de
Pernambuco, Recife, 2015.

MARONI, B. C.; STASI, L. C.; MACHADO, S. R.. Plantas medicinais do Cerrado de Botucatu. São Paulo: UNESP, 2006.

MARREIROS, N. A.; FERREIRA, E. C.; LUCENA, C. M.; PAIVA DE LUCENA, R. F.. Conhecimento botânico tradicional sobre plantas medicinais no Semiárido da Paraíba (Nordeste, Brasil). Ouricuri, v.5, n.1, p.110-144, 2015.

MARTINS, E. R.; CASTRO, D. M.; CASTELLANI, D. C.; DIAS, J. E.. Plantas medicinais. Viçosa: UFV, 2000.

MATOS, F. J. A.. Plantas da Medicina Popular do Nordeste: Propriedades Atribuídas e Confirmadas. Fortaleza: UFC, 1999.

MEDEIROS, M. F. T.; SENNA-VALLE, L.; ANDREATA, R. H. P. Histórico e o uso da 'salsa parrilha' (Smilax spp.) pelos boticários no Mosteiro de São Bento. Revista Brasileira de Biociências, Porto Alegre, v.5, n.1, p.27-29, 2007.

MEIRA, M.; SILVA, E. P.; DAVID, J. M.; DAVID, J. P.. Review of the genus Ipomoea: traditional uses, chemistry and biological activities. Brazilian Journal of Pharmacognosy, v.22, n.3, p.682-713, 2012.

MESSIAS, M. C. T. B.; MENEGATTO, M. F.; PRADO, A. C. C. SANTOS, B. R.; GUIMARÃES, M. F. M.. Uso popular de plantas medicinais e perfil socioeconômico dos usuários: um estudo em área urbana em Ouro Preto, MG, Brasil. Revista Brasileira de Plantas Medicinais, Campinas, v.17, n.1, p.76104, 2015.

MORALES, J. F.. Systematics of the tribe Echiteae and the genus Prestonia (Apocynaceae, Apocynoideae). Universität Bayreuth genehmigten Dissertation, 2017.

MOREIRA, A. L. C.. Jacquemontia Choisy (Convolvulaceae) nos estados de Goiás e Tocantins, Brasil: Estudos florísticos e taxonômicos. Dissertação (Mestrado em Botânica) Instituto de Ciências Biológicas da Universidade de Brasília, Brasília, 2014.

N'DANIKOU, S.; ACHIGAN-DAKO, E. G.. Corchorus aestuans L. Record from PROTA4U. In: BRINK, M.; ACHIGAN-DAKO, E. G.. PROTA: Plant Resources of Tropical. Wageningen, 2011.

NARDELLI, R.. Para Saber: 100 plantas Medicinais: A sabedoria da Natureza. Belém, 2005.

NAS. Agroforestry in the West African Sahel. Washington: National Academy of Sciences, 1984.

NASCIMENTO, A. V.. Série Plantas: Sempre-viva (Alternanthera tenella). 2014

NASCIMENTO, J. M.; CONCEIÇÃO, G. M.. Plantas medicinais e indicações terapêuticas da comunidade quilombola Olho d'água do Raposo, Caxias, Maranhão, Brasil. Revista de Biologia e Farmácia, v.6, n.2, p.138-151, 2011.

NETO, F. R. G.; ALMEIDA, G. S. S. A.; JESUS, N. G.; FONSECA, M. R.. Estudo Etnobotânico de plantas medicinais utilizadas pela Comunidade do Sisal no município de Catu, Bahia, Brasil. Revista Brasileira de Plantas Medicinais, Campinas, v.16, n.4, p.856-865, 2014.

OLIVEIRA, F. C.; ALBUQUERQUE, U. P.; FONSECA-KRUEL, V. S.; HANAZAKI, N.. Avanços nas pesquisas etnobotânicas no Brasil. Acta Botanica Brasilica, v.23, p.590-605, 2009.

OLIVEIRA, L. B.; CAVALCANTE, A. C. R.; MESQUITA, T. M. O.; ALVES, M. M. A.; SOUZA, E. B.; LEITE, E.. Identificação e 
Compatibilidade de Espécies Herbáceas Nativas e BRS Piatã (Brachiaria Brizantha Cv. Piatã). Sobral: EMBRAPA, 2015.

OLIVEIRA, P. V.; FERREIRA JUNIOR, J. C.; MOURA, F. S.; LIMA G. S.; OLIVEIRA, F. M.; OLIVEIRA, P. E.; CONSERVA, L. M.; GIULIETTI, A. M.; LEMOS, R. P.. Larvicidal activity of 94 extracts from ten plant species of northeastern of Brazil against Aedes aegypti L. (Diptera: Culicidae). Parasitology Research, v.107, p.403-407, 2010. DOI: https://10.1007/s00436-010-1880-4

OLIVEIRA, P. V.. Constituintes Químicos e Atividades Larvicida, Antioxidante e Antimicrobiana de Rourea doniana Baker (Connaraceae). Dissertação (Mestrado em Ciências Exatas) - Universidade Federal de Alagoas, Maceió, 2011.

OMOTAYO, F. O.; BOROKINI, T. I.. Comparative phytochemical and ethnomedicinal survey of selected medicinal plants in Nigeria. Scientific Research and Essays, v.7, n.9, p.989-999, 2012.

PANDA, A.; MISRA, M. K.. Ethnomedicinal survey of some wetland plants of South Orissa and their conservation. Ind. J. Trad. Knowl, v.10, n.2, p.296303, 2011.

PATEL, T.; JAIN, V.; DODIA, R.. Oldenlandia corymbosa L.: A Phytopharmacological review. International Journal of Phytopharmacy, v.4, n.3, p.79-82, 2014.

PAVITHRA, P. S.; SREEVIDYA, N.; VERMA, R. S.. Antibacterial and antioxidant activity of methanol extract of Evolvulus nummularius. Indian Journal of Pharmacology, v.41, p.233236, 2009

PEREZ, B. E. E.; CASASOLA, P. M.. Plantas medicinales de la matamba y el Piñonal, municipio de Jamapa, Veracruz. Instituto de Ecologia A. C. (INECOL), 2015.

PESAVENTO, F.. O azul fluminense: um estudo sobre o comércio do anil no Rio de Janeiro Colonial. Revista Econômica, v.7, n.2, p.207-231, 2005.

PILLA, M. A. C.; AMOROZO, M. C.; FURLAN, A.. Obtenção e uso das plantas medicinais no distrito de Martim Francisco município de Mogi-Mirim, SP, Brasil. Acta Botânica Brasilica v.20, p.789-802, 2006.

POTT, A.; POTT, V. J.. Plantas do Pantanal. Corumbá: EMBRAPA, 1994

PPG IV. A community-derived classification for extant lycophytes and ferns. Journal of Systematics and Evolution, v.54, n.6, p.563-603, 2016

RAMOS, G. G.; PINTO, M. G. C.; BAKKE, E. A.. Levantamento Etnobotânico de Mata Ciliar em Comunidade Ribeirinha do Município de Patos, Semiárido Nordestino. In: CONIDIS CONGRESSO INTERNACIONAL DA DIVERSIDADE DO SEMIÁRIDO, 2. Anais. 2018. p.1-6.

RANDAU, K. P.; FLORÊNCIO, D. C.; FERREIRA, C. P.; XAVIER, H. S.. Estudo farmacognóstico de Croton rhamnifolius H.B.K. e Croton rhamnifolioides Pax \& Hoffm. (Euphorbiaceae). Revista Brasileira da Farmacognosia, v.14, n.2, p.89-96, 2004.

REIS, C. S.; CONCEIÇÃO, G. M.. Aspectos Florísticos de um Fragmento de Vegetação, localizado no Município de Caxias, Maranhão, Brasil. Scientia Plena, v.6, n.2, p.1-17, 2010.

RITTER, M. R.; SOBIERAJSKI, G. R.; SCHENKEL, E. P.; MENTZ L. A.. Plantas usadas como medicinais no município de Ipê, RS, Brasil. Revista Brasileira de Farmacognosia, v.12, n.2, p.51-62, 2002. DOI: http://dx.doi.org/10.1590/S0102-
$695 \times 2002000200001$

RODRIGUES, K. A. F.; DIAS, C. N. D.; FLORÊNCIO, J. C.; VILANOVA, C. M.; GONÇALVES, J. R. S.; COUTINHO-MORAES, D. F.. Prospecção Fitoquímica e atividade Moluscicida de folhas de Momordica charantia L. Cad. Pesq., São Luís, v.17, n.2, 2010.

RODRIGUEZ, E.; TWERS, G. H. N.; MITCHELL, J. C.. Biological activities of sesquiterpene Lactones. Phytochemistry, v.15, p.1573-1580, 1976.

SAMOISY, A. K.; MAHOMOODALLY, M. F..

Ethnopharmacological analysis of medicinal plants used against non-communicable diseases in Rodrigues Island, Indian Ocean. Journal of Ethnopharmacol, v.173, n.15, p.2038, 2016.

SADRAEIAN, M.; GUIMARÃES, F. E. G.; ARAÚJO, A. P. U.; WORTHYLAKE, D. K.; LECOUR, L. J.; PINCUS, S. H.. Selective cytotoxicity of a novel immunotoxin based on pulchellin $A$ chain for cells expressing HIV envelope. Scientific Reports, v.7, n.7579, p.12, 2017.

SANTOS, A. C. B.; SILVA, M. A. P.; SANTOS, M. A. F.; LEITE, T. R.. Levantamento etnobotânico, químico e farmacológico de espécies de Apocynaceae Juss. Ocorrentes no Brasil. Revista Brasileira de Plantas Medicinais, v.15, n.3, p.442-458, 2013.

SARAIVA, I. R.; SOUSA, V. C.; SILVA, M. G. V.; MONTEIRO, J. A.; MORAIS, S. M.; FERREIRA JUNIOR, J. M.. Análise fitoquímica e avaliação das atividades leishmanicida, antioxidante e toxicidade frente à Artemia salina em extratos de Senna cana. In: CONGRESSO BRASILEIRO DE ENGENHARIA QUÍMICA, 21. Anais. Fortaleza, 2016.

SHARMA, V.; REDDY, K. R. C.; GAUTAM, D. S.

Phytochemistry and pharmacology of sida spinosa. International Journal of Green Pharmacy, v.12, p.07, 2018.

SILVA, G. A.; AKISUE, G.; OGA, S.. Ensaios Farmacológicos de Ação Hipoglicemiante dos extratos de Cissus sicyoides (L.) (insulina). In: SIMPÓSIO DE PLANTAS MEDICINAIS DO BRASIL, 13. Anais. Fortaleza, 1994.

SILVA, L. C.; PEGORARO, K. A.; PEREIRA, A. V.; ESMERINO, L. A.; CASS, Q. B.; BARISON, A.; BELTRAME, F. L.. Antimicrobial Activity of Alternanthera brasiliana Kuntze (Amaranthaceae): a Biomonitored Stud. Latin American Journal of Pharmacy, v.30, n.1, p.147-53, 2011

SILVA, C. G.; MARINHO, M. G. V.; LUCENA, M. F. A.; COSTA, J. G. M.. Levantamento etnobotânico de plantas medicinais em área de Caatinga na comunidade do Sítio Nazaré, município de Milagres, Ceará, Brasil. Revista Brasileira de Plantas Medicinais, v.17, n.1, p.133-142, 2015.

SILVA, R. B. L.. A etnobotânica de plantas medicinais da comunidade quilombola de Curiaú, Macapá - AP. Brasil. Dissertação (Mestrado em agronomia) - Universidade Federal Rural da Amazônia, Manaus, 2002.

SIMÕES, C. M. O.; SCHENKEL, E. P.; GOSMANN, G.; MELLO, J. C. P.; MENTZ, L. A. PETROVICK, P. R.. Farmacognosia: da planta ao medicamento. 5 ed. Porto Alegre: UFRGS; Florianópolis: UFSC, 2003.

SOUZA, F. A.; NETO, G. G.. Aspectos botânicos e de usos de Cissus verticillata (L.) Nicholson \& C. E. Jarvis (Vitaceae): insulina-vegetal. Flovet, v.1, p.21-39, 2009.

SOUZA, R. K. D.; MENDONÇA, A. C. A. M.; SILVA, M. A. P.. Aspectos etnobotânicos, fitoquímicos e farmacológicos de espécies de Rubiaceae no Brasil. Revista Cubana de Plantas Medicinales, v.18, n.1, p.140-156, 2013. 
SOUZA, V. C.; GIULIETTI, A. M.. Levantamento das espécies de Scrophulariaceae sensu lato nativas do brasil. Pesquisas, Botânica № 60:7-288. São Leopoldo: Instituto Anchietano de Pesquisas, 2009.

SUBIN, M. P.; REGHU, N.. Phytochemical Screening and Antibacterial Properties of Croton hirtus L'Her. Plant Against Some Important Pathogenic Bacteria. Nature Environment and Pollution Technology, v.11, n.1, p.59-64, 2012.

TEIXEIRA, G.; MACIEL, S.; PIETROBOM, R.. Potencial utilitário de licófitas e samambaias: aplicabilidade ao contexto amazônico. Macapá, Biota Amazônia, v.5, n.1, p.68-73, 2015.

TONA, L.; NGIMBI, N. P.; TSAKALA, M.; MESIA, K.; CIMANGA, K. S.; APERS.. Antimalarial activity of 20 crude extracts from nine African medicinal plants used in Kinshasa, Congo. Journal of Ethnopharmacol, v.68, p.193-203, 1999.

ULLOA, U. C.; ACEVEDO-RODRÍGUEZ, P.; BECK, S.; BELGRANO, M. J.; BERNAL, R.; BERRY, P. E.; BRAKO, L.; CELIS, M.; DAVIDSE, G.; FORZZA, R.C.; GRADSTEIN, S.R.; HOKCHE, O.; LEÓN, B.; LEÓN-YÁNEZ, S.; MAGILL, R.E.; NEILL, D.A.; NEE, M.; RAVEN, P.H.; STIMME, H.; STRONG, M. T.; VILLASEÑOR, J. L.; ZARUCCHI, J.L.; ZULOAGA, F. O.; JØRGENSEN, P. M.. An integrated assessment of the vascular plant species of the Americas. Science, v.358, p.1614-1617, 2017.

VÁSQUEZ, S. P. F.; MENDONÇA, M. S.; NODA, S. N.. Etnobotânica de plantas medicinais em comunidades ribeirinhas do Município de Manacapuru, Amazonas, Brasil. Acta Amazônica, v.44, n.4, p.457-472, 2014.

VENDRUSCOLO, G. S.; SIMÕES, C. M. O.; MENTZ, L. A. Etnobotânica no Rio Grande do Sul: análise comparativa entre o conhecimento original e atual sobre as plantas medicinais nativas. Pesquisas, Botânica no56. São Leopoldo: In stituto Anchietano de Pesquisas, 2005.
VIEIRA, L. S.. Fitoterapia da Amazônia, Manual de Plantas Medicinais: a farmácia de Deus. 2 ed. São Paulo: Agronômica Ceres, 1992.

VIEIRA, R. F.; MARTINS, V. M.. Recursos Genéticos de Plantas Medicinais do Cerrado: uma compilação de dados. Revista Brasileira de Plantas Medicinais, Botucatu, v.3, n.1, p.13-36, 2000.

WANG, H.; YANG, J.-X.; LIU, G.-Y.; LOU, J.; LI, L.; HU, Q.; GAO, $X .-M$.. Editorial committee of fujian institute of chinese medicine. Fujian Materia Medica. Fujian Science and Technology Press, Fuzhou, v.27, n.6, p.205-332, 1994.

YAHARA, T.; JAVADI, F.; ONODA, Y.; QUEIROZ, L. P.; FAITH, D. P.; PRADO, D. E.; AKASAKA, M.; KADOYA, T.; ISHIHAMA, F.; DAVIES, S.; SLIK, J. W. F.; YI, T.; MA, K.; BIN, C.; DARNAEDI, D.; PENNINGTON, R. T.; TUDA, M.; SHIMADA, M.; ITO, M.; EGAN, A. E.; BUERKI, S.; RAES, N.; KAJITA, T.; VATANPARAST, M.; MIMURA, M.; TACHIDA, H.; IWASA, Y.; SMITH, G. F.; VICTOR, J. E.; NKONKI, T.. Global legume diversity assessment: Concepts, key indicators, and strategies. Taxon, v.62, p.249266, 2013.

ZAFAR, R.. Medicinal plants of India. New Delhi: CBS Publishers and Distributor, 1994.

ZAYED, M. Z.; SALLAM, S. M. A.; SHETTA, N. D.. Review Article on Leucaena leucocephala As One of The Miracle Timber Trees. International Journal of Pharmacy and Pharmaceutical Sciences, v.10, n.1, p.1-7, 2018. DOI: http://10.22159/ijpps.2018v10i1.18250

ZAYED, M.; BENEDICT, S.. Phytochemical constituents of the leaves of Leucaena leucocephala from malaysia. International Journal of Pharmacy and Pharmaceutical Sciences, v.8, p.174-9, 2016. DOI: http://10.22159/iipps.2016v8i12.11582

A CBPC - Companhia Brasileira de Produção Científica (CNPJ: 11.221.422/0001-03) detém os direitos materiais desta publicação. Os direitos referem-se à publicação do trabalho em qualquer parte do mundo, incluindo os direitos às renovações, expansões e disseminações da contribuição, bem como outros direitos subsidiários. Todos os trabalhos publicados eletronicamente poderão posteriormente ser publicados em coletâneas impressas sob coordenação da Sustenere Publishing, da Companhia Brasileira de Produção Científica e seus parceiros autorizados. Os (as) autores (as) preservam os direitos autorais, mas não têm permissão para a publicação da contribuição em outro meio, impresso ou digital, em português ou em tradução. 